\title{
High-Content Analysis of MicroRNAs Facilitates the Development of Combinatorial Therapies for Vascular Diseases
}

\section{Jian Zhang}

University of Heidelberg

Vytaute Starkuviene

University of Heidelberg

Holger Erfle

University of Heidelberg

Zhaohui Wang

University of Heidelberg

Manuel Gunkel

University of Heidelberg

Carsten Sticht

University of Heidelberg

Kejia Kan

University of Heidelberg

Norbert Gretz

University of Heidelberg

Nuh Rahbari

University of Heidelberg

Michael Keese ( $\square$ Michael.Keese@umm.de)

University of Heidelberg

\section{Research Article}

Keywords: VSMC, cardiovascular diseases, microRNA, contractile phenotype, synthetic phenotype, phenotypic switch

Posted Date: May 12th, 2021

DOl: https://doi.org/10.21203/rs.3.rs-504665/v1

License: (c) (1) This work is licensed under a Creative Commons Attribution 4.0 International License.

Read Full License 



\section{development of combinatorial therapies for vascular diseases}

running title: identification of miRNAs regulating phenotypic switch

Jian Zhang a,b, Vytaute Starkuviene ${ }^{\text {b,c, }{ }^{\text {, }} \text {, Holger Erfle }}{ }^{b}$, Zhaohui Wang ${ }^{a, b}$, Manuel Gunkel ${ }^{b}$, Carsten Sticht ${ }^{\text {d, Kejia Kan }}{ }^{a}$, Norbert Gretz ${ }^{d}$, Nuh Rahbaria, Michael Keese ${ }^{a}$, a Chirurgische Klinik and European Center of Angioscience (ECAS), Medical Faculty Mannheim, University of Heidelberg, Mannheim, Germany

b BioQuant, University of Heidelberg, Heidelberg, Germany

c Institute of Biosciences, Vilnius University Life Sciences Center, Vilnius, Lithuania

d Medical Research Center, Medical Faculty Mannheim, University of Heidelberg, Mannheim, Germany

* Corresponding authors: Michael Keese and Vytaute Starkuviene equally contribute to corresponding authors. Michael Keese, E-mail: Michael.Keese@umm.de and Vytaute Starkuviene, E-mail:

vytaute.starkuviene@bioquant.uni-heidelberg.de

\section{Abstract}

In response to vascular injury vascular smooth muscle cells (VSMCs) alternate between a differentiated (contractile) and a dedifferentiated (synthetic) state or phenotype. Although parts of the signaling cascade regulating the phenotypic switch have been described, little is known on the role of miRNAs involved. To systematically address this issue, we have established a microscopy-based quantitative assay and identified 23 miRNAs that induced contractile phenotypes when over-expressed. These were then correlated to miRNAs identified from RNA-sequencing when comparing cells in the contractile and synthetic states. Using both approaches, six miRNAs (miR-132-3p, miR138-5p, miR-141-3p, miR-145-5p, miR-150-5p, and miR-22-3p) were filtered as 
candidates that induce the phenotypic switch from synthetic to contractile. To identify

27 potentially common regulatory mechanisms of these six miRNAs, their predicted targets were compared with five miRNAs sharing ZBTB20, ZNF704, and EIF4EBP2 as common potential targets and four miRNAs sharing 16 common potential targets. The interaction network consisting of these 19 targets and additional 18 hub targets were created to facilitate validation of miRNA-mRNA interactions by suggesting the most plausible pairs.

32 Furthermore, the information on drug candidates was integrated into the network to predict novel combinatorial therapies that encompass the complexity of miRNAs-mediated regulation. This is the first study that combines phenotypic screening approach with RNA sequencing and bioinformatics to systematically identify miRNAs-mediated pathways and to identify potential drug candidates to positively influence the phenotypic switch of 37 VSMCs.

Keywords: VSMC, cardiovascular diseases, microRNA, contractile phenotype, synthetic phenotype, phenotypic switch

\section{Abbreviations}

CSI: Cell Shape Index, E: Elongation, FC: Fold change, FCS: Fetal Calf Serum, HAoVSMCs: Human Aortic Vascular Smooth Muscle Cells, KNIME: the Konstanz Information MinEr, KOBAS: KEGG Orthology Based Annotation System, PAD: peripheral artery disease, Ratio of con/syn: Ratio of contractile/synthetic, SM22-a: smooth muscle 22- $\alpha$, SmGM-2: Smooth Muscle Cell Growth Medium-2, SM-MHC: Smooth Muscle Myosin

46 Heavy Chain, TGF- $\beta$ : Transforming Growth Factor- $\beta$, $\alpha$-SMA: $\alpha$-smooth muscle actin, VSMC: vascular smooth muscle cells. 
Cardiovascular disease is a leading cause of death worldwide and accounts for more than 17.3 million deaths per year, with an estimated increase in incidence to 23.6 million by 2030ำ. Peripheral artery disease, atherosclerosis after coronary artery disease and stroke

52 are the most common manifestations ${ }^{2}$. Vascular smooth muscle cells (VSMCs) frequently account for related pathophysiological processes within the blood vessel wall. Unlike many other mature cell types in the adult body, VSMCs do not terminally differentiate but retain a remarkable plasticity ${ }^{3}$. Predominantly, they are found in two principal phenotypes: contractile and synthetic ${ }^{4}$. Under normal physiological conditions the cells maintain the

57 muscular tone of blood vessels and acquire the quiescent/contractile phenotype. In response to vascular injuries or alterations in the local environment, contractile VSMCs can re-enter the cell cycle, undergo a phenotypic switch to the synthetic phenotype and drive the progression of vascular diseases ${ }^{3}$. The phenotype switch facilitates plaque formation, which is the prerequisite for atherosclerosis ${ }^{5}$. It is established by now, that

62 inhibiting VSMCs phenotypic switching may be beneficial in advanced stages of this 63 disease.

64 The cells in the quiescent/contractile phenotype show low levels of migration and proliferation. Morphologically, the contractile VSMCs display a fusiform or spindle-like shape, abundant myofilaments and a heterochromatic nucleus ${ }^{6}$. In contrast, the synthetic VSMCs adopt a rhomboid shape without specific filamentous cytoplasm, but with the extensive rough endoplasmic reticulum, Golgi complex, and a euchromatic nucleus ${ }^{6,7}$. The phenotype switch is tightly regulated on a molecular level. Oxidative stress, autophagy, the expression levels and repertoire of matrix metalloproteinases and integrins have been

71 shown to play important roles in the phenotypic switch and, consequently, vascular 
remodeling ${ }^{8}$. For instance, the serum response factor acts directly or indirectly on most VSMC contractile genes ${ }^{9}$. Furthermore, a recent study shows that fibroblast growth factor 12 (FGF12) reduces cell proliferation through the p53 pathway and up-regulates key factors related to the differentiation of the VSMC lineage, such as myocardin and serum response factors ${ }^{10}$. At any rate, when acquiring one of these phenotypes, VSMCs alter VSMC phenotypic modulation ${ }^{11}$.

81 Phenotypic switch is also regulated post-transcriptionally by microRNAs (miRNAs), that are endogenous short non-coding RNAs containing 21 23 nucleotides ${ }^{12}$. Several miRNAs are already proved to play essential roles in the modulation of VSMCs' function and phenotypic switch in vitro and/or in animal models ${ }^{13-17}$. Yamaguchi et al. ${ }^{14}$ stated that miR145 could induce a morphological change in VSMCs from a rhomboid- to a spindle-like shape in human ES-pre-SMCs. Furthermore, overexpression of miR-145 promoted differentiation and inhibited the proliferation of cultured VSMCs ${ }^{13,18}$. Platelet-Derived 88 Growth Factor (PDGF), a potent stimulator of VSMCs' migration, can downregulate the expression of miR-145, inducing podosome formation. This appears to be mediated 90 through the activity of Src and $p 53^{19}$. miR-145-deficient VSMCs, on the other hand, failed to demonstrate a contractile phenotype in response to vasopressin stimuli ${ }^{20}$, indicating a context-dependent role of miRNAs. Huang et al. ${ }^{15}$ showed that miR-22 mimics significantly reduced proliferation and migration of (VSMCs) via targeting of methyl CpG-binding protein $2(\mathrm{MECP} 2)^{21}$. In another example, Afzal et al. ${ }^{16}$ revealed that overexpression of 95 miR-214 in VSMCs significantly decreased proliferation and migration via downregulation 
of NCK-associated protein 1 (NCKAP1) expression, which in turn diminished lamellipodia

97 formation ${ }^{22}$. Pan et al. ${ }^{17}$ found that miR-663a was significantly downregulated in VSMCs 98 after PDGF treatment, whereas its expression markedly increased during cell differentiation. Furthermore, it was demonstrated that overexpression of miR-663a 100 increased expression of VSMC differentiation marker genes such as SM22 $\alpha$, SMA- $\alpha$, calponin, and SM-MHC, which potently inhibit PDGF-induced proliferation and migration.

102 Furthermore, recent reports suggest that miRNA application may be a potentially effective 103 therapy. For example, Yang et al. applied in a wire-injury mouse model locallyof AgomiR22 or miR-22 inhibitor to demonstrate the modulation of the switch phenotype ${ }^{23}$.

105 Despite the above-mentioned examples, there is still a fairly little number of miRNAs known to induce the phenotypic switch ${ }^{24}$. In order to efficiently and systematically identify such molecules, large-scale methods were recently successfully harnessed. For instance, expression profiling of miRNAs ${ }^{25}$ and circular $\mathrm{RNAs}^{26}$ or miRNA sequencing ${ }^{27}$ discovered a number of yet unknown regulators. High-content microscopy-based screening, despite being tedious, is clearly the method of choice as it directly provides information about the cell morphology. Previously, RNAi screening of protein-coding genes ${ }^{28}$ was performed and, by quantifying the changes in proliferation and migration of human aortic vascular smooth muscle cells (HAoVSMCs), identified 23 genes involved in the phenotypic switch ${ }^{28}$.

114 In this study, we have established a high-content analysis platform to identify and quantify contractile and synthetic phenotypes in cell populations. Our work can be easily upscaled to genome-level studies and opens the possibility for deeper understanding of the

117 functions of miRNAs in the regulation of VSMC's differentiation and phenotypic switch, 118 providing new insights

119 into the mechanisms of vascular development, function, and dysfunction. 


\section{Materials and Methods}

\section{Cell culture}

122

123

124

125

126

127

The HAoVSMCs were cultured in Smooth Muscle Cell Growth Medium-2 (SmGM-2) supplemented with $5 \%$ fetal calf serum (FCS), $0.1 \%$ epidermal growth factor (EGF), $0.1 \%$ basic fibroblast growth factor (bFGF) and $0.1 \%$ Insulin (all reagents and cells were purchased from Promocell, Germany). Cells were incubated in a humidified incubator at $37^{\circ} \mathrm{C}$ with $5 \% \mathrm{CO}_{2}$. The cells were used between passage 4 and 9 in accordance with our institutional guidelines for research on human tissues and cells. A mycoplasma test was performed regularly (at least once a month) and the cells were checked daily under a microscope. The freshly prepared cryopreservation medium contained $10 \%(\mathrm{v} / \mathrm{v})$ dimethyl sulfoxide (DMSO) and 10\% FCS dissolved in SmGM-2 medium. For starvation, the cells were cultured in $1 \%$ FCS for $48 \mathrm{~h}$.

\section{Immunofluorescence}

At 48h after seeding, the HAoVSMCs were fixed with 3\% paraformaldehyde (PFA) for 15 min, permeabilized with $0.1 \%$ Triton-X 100 in PBS for $10 \mathrm{~min}$, and the background was blocked with $1 \%$ BSA in PBS for $1 \mathrm{~h}$ before staining with primary and secondary antibodies. The primary antibody for SM $\alpha$-actin (1:200, Rabbit anti-alpha-actin, Abcam, UK) was diluted with blocking buffer and incubated overnight at $4^{\circ} \mathrm{C}$. The secondary goat-anti-rabbit antibody (1:1000, Life Technologies, USA) conjugated with AlexaFluor 488 was diluted with the blocking buffer and incubated for $1 \mathrm{~h}$ at RT. Then, TOTO $(642 / 660,1: 1000$, Invitrogen, Germany) was added to the secondary antibody solution for nuclear staining (incubation for 30 min at RT) in accordance with the manufacturer's protocol. 
143 For the preparation of plates for reverse solid phase transfection in the multi-well plates, 144 siRNA/miRNA transfection solution was dispensed on 96- and 384-well plates (BD 145 Biosciences / Costar SIGMA, Germany) using a Microlab STAR pipetting robot (Hamilton, 146 Reno, NV, USA) ${ }^{29}$. In brief, $3 \mu \mathrm{L}$ OptiMEM (Invitrogen, Darmstadt, Germany) containing 0.4 M sucrose was transferred to each well of a 384-well plate. Then, 3.5 $\mu \mathrm{L}$ Lipofectamine 2000 (Thermo Fischer Scientific, Waltham, USA) was added. After that, $5 \mu \mathrm{L}$ of the respective siRNA/miRNA stock solution $(3 \mu \mathrm{M})$ followed by $7.25 \mu \mathrm{L}$ of a $0.2 \%(\mathrm{w} / \mathrm{v})$ gelatin solution containing $1 \times 10-2 \%(v / v)$ fibronectin (Sigma-Aldrich, Taufkirchen, Germany) were added and mixed thoroughly. For 384-well plates, the transfection solution was diluted with $\mathrm{H}_{2} \mathrm{O}$ in the ratio of $1: 10$ and $5 \mu \mathrm{L}$ of the diluted transfection solution was added to each well. The HAoVSMCs were seeded in the pre-coated 384-well plates at a density of $400-500$ cells per well in $60 \mu \mathrm{L}$ of culture medium per well and incubated for $72 \mathrm{~h}$. Cells were stained with Hoechst $33342(0.2 \mu \mathrm{g} / \mathrm{mL}$, Invitrogen, Germany) to label nuclei and DilC12(3) (2 $\mu \mathrm{g} / \mathrm{mL}$, Invitrogen, Germany) was used to label the whole cell body. The list of miRNAs is provided in Supplemental Table 1.

\section{Microscopy and image processing}

159 Cells were observed time-lapse under a fluorescence microscope (Olympus IX81, 10x objective) for imaging. Photographs of the samples were taken dynamically using a 161 fluorescence microscope at intervals of $24 \mathrm{~h}, 48 \mathrm{~h}$, and $72 \mathrm{~h}$. The low magnification (4x) objective (Olympus inverted microscope CKX41) was used for the wound healing assay.

163 The images were preprocessed by Fiji-imageJ software (National Institutes of Health, USA) and imported into the Konstanz Information MinEr software (KNIME, www.knime.org) for 
determining the morphological parameters $(\mathrm{E}=$ major axis/minor axis) and $(\mathrm{CSI}=$ $4^{*} \pi^{*}$ area/perimeter $\left.{ }^{2}\right)^{30}$ of the HAoVSMCs using its customized workflow (Supplemental

Figure 1). The optimizations for the workflow, including picture background optimization, cell image segmentation, gray value calculation, cell morphology comparison, cell phenotyping parameter calculation and classification were performed throughout the analysis. When optimized internal parameters fitted with the photos, the result was generated. For the immunofluorescence experiments, the expression intensity and percentage of positive cells were determined by counting the number of differentiated cells 173 in 10 fields of view for each group.

\section{RNA sequencing}

After being seeded in the T25 flasks, the HAoVSMCs were treated with the media containing low and normal amounts of FCS then they were incubated for $48 \mathrm{~h}$. Then the HAoVSMCs were harvested, transferred into RNAlater ${ }^{T M}$ RNA Stabilization Reagent (Qiagen, Germany) and homogenized immediately. MiRNAs were isolated using the Allprep RNA isolation kit (Qiagen, Germany) in accordance with the manufacturer's protocol and analyzed using Agilent Bioanalyzer 2100 Expert (B.02.08.SI648, Agilent, Santa Clara, CA, USA). After the RNA integrity number (RIN) of miRNA had been identified, the RNA samples were sent to the Beijing Genomics Institute (BGI, Shenzhen, China) for miRNA detection and sequencing service. Filtered miRNAs were quantified per library by realigning reads of at least $17 \mathrm{bp}$ length to predicted miRNAs in QuickMIRSeq ${ }^{31}$. QuickMIRSeq extensively filters the data by joint mapping to the transcriptome and ribosomal RNA to reduce false positives. Sequences were aligned to the reference genome GRCh38.p13. The count data was transformed to log2-counts per million (logCPM) using the voom-function from the limma package ${ }^{32}$ in R. Differential expression 
analysis was performed using the limma package. A false-positive rate of $\alpha=0.05$ with

190 FDR correction was taken as the level of significance. Volcano plots and heatmaps were created using ggplot2 package (version 2.2.1) and the complex Heatmap ${ }^{33}$ (version 2.0.0).

\section{Statistical and bioinformatics analysis}

All the genes derived from the PubMed searching were subjected to analysis using the miRWalk database (version 3.0 $)^{34}$. The data obtained from established cell lines are presented as means $\pm S D$ from at least three separate experiments, which were performed at least in triplicate. Statistical analysis was carried out using one-way analysis of variance (ANOVA) followed by Bonferroni post hoc for multiple groups or Student's ttest between two groups. For the immunofluorescence experiments, we described the expression intensity and percentage of positive cells by counting the number of differentiated cells in 10 fields of view for each group.

Then, we used Drug-Gene Interaction Database (DGldb) (http://www.dgidb.org/), including several drug databases (DrugBank, PharmGKB, ChEMBL), clinical trial databases and literature from PubMed ${ }^{35}$. The top 3 hub target genes of each miRNA were selected from the protein-protein interaction analysis in STRING database (www.string$\mathrm{db}$. org) with the maximal clique centrality (MCC) method using cytoHubba plugin software in Cytoscape ${ }^{36}$. Then, we imported these target hub genes into DGldb to explore existing drugs or small organic compounds. Results were displayed using the R packages ggplot2 (version 3.2.1) and ggalluvial (version 0.11.1). Meanwhile, target gene sets of the hit miRNAs were enriched by Gene Set Enrichment Analysis (GSEA) algorithm and KEGG Orthology-Based Annotation System (KOBAS) database (version 3.0). $p<0.05$ was considered statistically significant (IBM SPSS Statistics 20). ${ }^{*} p<0.05,{ }^{* *} p<0.01$. 
213 Establishment of quantitative microscopy-based switch assay

214 VSMCs undergo phenotypic changes when stimulated by environmental changes.

215 Phenotypic change may be induced by low serum stimulation, leading the cells to change 216 from the synthetic to the contractile state 37,38 . In our study, we used HAoVSMCs which 217 were isolated from plaque-free regions of the human aorta to model the behavior of

218 VSMCs. For induction of the contractile state cells were incubated in the growth medium 219 containing 5\% FCS (see Materials and Methods). The cells stained positive for $\alpha$-SMA 220 under these conditions. Initially, we have tested whether changing FCS concentration in 221 the growth medium to $1 \%$ would induce contractile phenotype as expected ${ }^{37,38}$. Indeed, 222 cells incubated in 1\% FCS for 48h displayed higher intensity of a-SMA specific signal than 223 the control group (Fig. 1A). We next tested the migratory potential of cells with the induced 224 contractile phenotype. Following their incubation in low serum media for 48h, wounding 225 with a $20 \mu \mathrm{L}$ pipette tip was done and the gap closure was monitored after $24 \mathrm{~h}$. Wound 226 closure after serum starvation was significantly inhibited. In contrast, HAoVSMCs cultured 227 in the high serum medium (10\% FCS) showed an obvious increase in migration as 228 compared to the control group (5\% FCS) (Fig. 1B, C).

As cells in the contractile state were elongated (Fig. 1A), we subsequently undertook a 230 quantitative study of the morphology changes. The 384-well plates were fixed with $3 \%$ 231 PFA and stained with Hoechst 33342 to label nuclei and DilC12(3) to label the whole cell 232 body via targeting the lipid bilayer of plasma membrane ${ }^{39}$. After staining with DilC12(3), 233 the background of the photographs had a robust signal of interference with limited 234 photobleaching and phototoxicity for optimal imaging. Hence, DilC12(3) was used for 30 
min when the cells were still in suspension before seeding. Artificial intelligence and

236 machine learning methods were applied to study the definition and threshold optimization

237 of elongation (E) and cell shape index (CSI) of cells cultured under normal or low serum

238 concentrations. During the learning phase, we taught the KNIME software how to 239 distinguish between the contractile and synthetic phenotype of HAoVSMCs and generated 240 an optimized workflow (Supplemental Fig.1). The larger the $E$ value (E $>1$ in the range)

241 is, the slender the cell appears. CSI values indicated how likely the cell morphology 242 resembled a closed circle. The larger the CSI (range $0<\mathrm{CSI}<1$ ) is, the closer the cell is 243 to a circle. Accordingly, $\mathrm{E}$ and $\mathrm{CSI}$ values were $\mathrm{E}>3$ and $0<\mathrm{CSI}<0.4$, for the contractile 244 phenotype, respectively; and $1<\mathrm{E}<3$ and $0.6<\mathrm{CSI}<1$, respectively for the synthetic 245 phenotype. In order to confirm that the KNIME could precisely recognize the different 246 phenotypes of HAoVSMCs, validation was performed by defining the cells manually 247 ( 1200 cells). By this, we could test and adapt this software to a high number of cells and 248 could evaluate and predict the precision and feasibility of the entire workflow.

249 In order to verify that the cell morphology can be used as an effective high-throughput 250 screening method to detect phenotypic switch, four miRNAs (miR-22-3p, miR-145-5p, 251 miR-214-3p, and miR-663a, (miR-22, miR-145, miR-214, and miR-663a in the following)) 252 that are known to effectively induce a phenotypic switch were used ${ }^{13-17}$. Images of the 253 transfected cells were taken at intervals of $24 \mathrm{~h}, 48 \mathrm{~h}$, and $72 \mathrm{~h}$ and analyzed in KNIME. 254 After segmentation, the cells were grouped to contractile, synthetic and undecided 255 phenotypic groups $(0.4<\mathrm{CSI}<0.6)$. Finally, the ratio of contractile / synthetic (Ratio of 256 con / syn) was calculated (Ratio = number of contractile / number of synthetic). Majority 257 of our test miRNAs significantly promoted the conversion of cells into contractile 258 phenotype as compared with the control group (Fig. 2). The ratios of con / syn in 
HAoVSMCs were increasing after transfection of miR-22, miR-145, miR-214, and miR-

260 663a. To summarize, we established an accurate, reliable, fast, and easy to apply 261 screening method based on cell morphology, which could be upscaled for screening 262 multiple miRNAs. Until now, the morphological parameters, such as CSI, have not been 263 used in any high-throughput screening approach.

miRNA overexpression screen

265

266

267

268

269

270

271

272

273

274

275

276

277

278

279

280

281

To obtain a list of genes closely related to the phenotypic switch of HAoVSMCs, an extensive literature search was performed using the following search terms: "human vascular smooth muscle cell, phenotypic switch and miRNA (or miR)" in PubMed, covering the last 10 years. As a result, 101 candidate genes with possible direct impact or relation to the phenotypic switch were identified. Then, all the 101 genes were subjected to an analysis using the miRWalk database (version 3.0$)^{34}$, and 87364 miRNA potential binding sites for these genes were obtained using the filtering options: 3'-UTR, TargetScan, miRDB, and miRtarbase. From those, 10154 miRNA-gene combinations with clearly evidenced effect in publications on HAoVSMCs were selected. After removing the duplicates, 1554 miRNAs were derived. Finally, by sorting the number of genes potentially influenced by these 1554 miRNAs, the top 50 miRNAs were selected for the screening according to the highest numbers of targeted genes. By this, we aim to validate and reconstruct the complex miRNA-mediated regulatory networks required for the phenotypic switch.

The oligoes for selected miRNAs over-expression were prepared (Dharmacon, CO, USA) (Supplemental Table 1). 384-well plates were pre-coated with these oligoes ${ }^{29,40-42}$ (three wells per oligo), HAoVSMCs were seeded and incubated for $72 \mathrm{~h}$. Then, the plates were 
fixed with 3\% PFA, stained with Hoechst 33342 and DilC12(3). Images were analyzed

283 using the KNIME software and E, CSI, and ratio of con / syn of cells in the treatment group and control group were calculated (Supplemental Fig 2). The heatmap (Supplemental

Fig 2A) shows the HAoVSMC phenotypic changes after miRNA transfection in each well of a 384-well plate (three independent experiments were performed and indicated as screen A, B, C). Two out of our three positive controls (miR-22 and miR-145) induced the contractile phenotype in all three replicates. We ranked the hits according to the averaged fold changes of their ratios of con / syn to that of the negative control (Supplemental Fig 2B). According to this ranking, the overexpression of 8 miRNAs (miR-138-5p, miR-1505p, miR-141-3p, miR-139-5p, miR-338-3p, miR-132-3p, miR-92a-3p and miR-130a-3p) induced a strong switch to the contractile phenotype in all three replicates (the averaged 293 fold change to the negative control is $>1.5$ ). Some of the recent studies match this finding, 294 for example: Chen et al. ${ }^{43}$ indicated that miR-150-5p may exert inhibitory effects on excessive proliferation and migration of pulmonary artery smooth muscle cells (PASMCs). Yet another 15 miRNAs induced a weaker transition (the averaged fold change > 1.2). 15 miRNAs led to a decrease in the averaged ratio of con / syn when over-expressed, 298 indicating that more cells remained in the synthetic state. Five of them (miR-148a-3p, miR15b-5p, miR-205-5p, miR-486-3p, and miR-93-5p) were strong hits with a decreased ratio 300 in all replicates. Our data correlates to the previously published work for some of these miRNAs; for instance, miR-93-5p is upregulated in proliferating rat HAoVSMCs both in 302 vivo and in vitro ${ }^{44}$ and miR-93-5p inhibitor prevented HAoVSMC proliferation and 303 migration in this study. Finally, 10 miRNAs induced no significant changes under these 304 conditions. 


\section{Validation of miRNAs that induce the contractile phenotype}

306 In order to validate miRNAs that induced the contractile phenotype when overexpressed

307 by the transient transfection, we carried out the miRNA sequencing in contractile 308 HAoVSMC. The HAoVSMC cells were collected under two conditions: the treatment group 309 with 1\% FCS, 48h incubation and the control group with 5\% FCS, 48h of incubation. For 310 each condition four individual replicates were collected. miRNAs were extracted and the

311 RNA integrity number (RIN) of miRNAs was determined and sequenced (Supplemental

312 Table 2). The overall information of these groups was shown in the heatmap of each

313 replicate, indicating closeness and the difference between the groups (Fig. 3A). When 314 averaged over all four replicates, 153 miRNAs were upregulated, and 143 miRNAs were 315 downregulated in the contractile cell group compared to the control group, as shown in 316 the volcano plot (Fig. 3B).

317 After comparing with the results of the microscopy-based screen and sequencing of 318 miRNAs, we found six overlapping miRNAs (Fig. 3C). All six molecules were hits in the 319 microscopy-based screen when overexpressed and shifted cells to contractile phenotype.

320 In agreement to that, they also were found at elevated levels in cells undergoing a switch 321 to contractile phenotype. Among the over-expressed miRNAs we could identify miR-22 322 and miR-145 that served as the robust positive controls for the microcopy-based analysis 323 (Fig. 2). Besides, we identified four other molecules that induced the contractile phenotype 324 when over-expressed (miR-132-3p, miR-138-5p, miR-141-3p, and miR-150-5p). The 325 quality of the data is underlined by the observation that all four miRNAs were quantified 326 as strong hits in microscopy-based switch assays (Supplemental Fig. 2). 
327 Furthermore, our hits have been identified as regulators of migration and proliferation in

328 the previous studies via targeting key regulators of gene expression or cell homeostasis, especially, that related to hypoxia-induced responses. For instance, Liu et al. ${ }^{45}$ indicated that overexpression of miR-138 reduced proliferation of human ASMCs (aortic smooth muscle cells), via targeting of 3'-UTR of PDK1 (pyruvate dehydrogenase kinase 1) mRNA.

332 Guo et al. ${ }^{46}$ suggested that overexpression of miR-145 significantly inhibited the 333 expression of CD40 and the differentiation of VSMCs, Chen et al. ${ }^{43}$ indicated that miR334150 may exert inhibitory effects on excessive proliferation and migration of PASMCs 335 through down-regulation of hypoxia-induced factor $1 \alpha$ (HIF-1 $\alpha)$.

\section{miRNA-mediated regulatory networks inducing the contractile phenotype}

337 Having identified promising candidate miRNAs, we next analyzed what common 338 regulatory mechanisms could potentially account for the induction of the contractile phenotype. Not only four top screen hits, but also our positive controls miR-22 and miR145 were considered as overlapping hit for this analysis (Fig. 3C). All six miRNAs belong to different miRNA seed families and we used miRWalk ${ }^{34}$ to extract the putative target genes for every molecule. In total, we obtained 3,497 putative target genes via miRWalk database for our 6 miRNAs (Supplementary Table 3). Of which, miRNA-145 ( $n=738$ ) and miRNA-150 ( $n=776$ ) have more target genes than others (data not shown). In order to cluster these targets to the regulatory networks, two online analytic software tools, $346 \mathrm{KOBAS}^{47}$ and Metascape ${ }^{48}$, were employed. Combining the results of the two analyses, a number of pathways already connected to the phenotypic switch of VSMC were

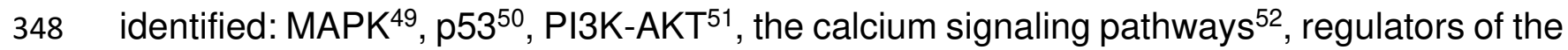
349 cell cycle ${ }^{53}$, TGF-beta signaling pathway ${ }^{54}$, or vascular smooth muscle contraction ${ }^{55}$, 
herewith demonstrating a high quality of our screens. More closely, we looked to the

351 putative regulators of cell adhesion to the extracellular matrix (ECM) due to their crucial

352 role in regulating cell shape and migration. For instance, there are studies demonstrating,

353 that controlling cell adhesion and shape via micropatterned surfaces, could restore the

354 contractile phenotype of VSMCs in vitro ${ }^{30}$. Indeed, 65 targets of these miRNAs are

355 regulators of focal adhesion formation and dynamics (e.g., ARF6, STX16 or PEAK1) with

356 over 40 predicted targets involved in the cell migration.

357

Next, we analysed whether six miRNAs share their targets and by this potentiate their

358 functional activity. There are no overlapped genes among all six miRNAs, however, even

359 three potential targets are shared among five miRNAs (Supplemental Table 4), despite

360 varying seed sequences. ZBTB20 (Zinc finger and BTB domain-containing protein 20),

361 ZNF704 (Zinc finger protein 704), and EIF4EBP2 (Eukaryotic translation initiation factor

4E-binding protein 2) are the proteins of broad functions in transcription and translation, respectively. No experimental validation data are available for these targets, therefore, further research is needed to test the possibility that switch regulating miRNAs mutually enhance their activities by targeting the same subset of mRNAs. Further 16 targets are shared among four miRNAs. Even six of them are transcription regulators (e.g., CREB1, phosphorylation dependent transcription factor), with other targets encoding regulators of signaling (e.g., MAP3K3 kinase) and protein modifications (e.g., ST3GAL6 sialyltransferase). So far, only two of them are validated by the strong methods including 370 luciferase reporter assay, Western blot, and qRT-PCR (CDK6 ${ }^{56}$ as a target of miR-145 371 and CREB1 ${ }^{57}$ as a target of miR-150). Therefore, more work is ahead for validation of the 372 remaining predicted miRNA-mRNA interactions. Finally, 83 potential targets were shared 373 by three miRNAs out of six analysed. The group is largely comprised of regulators of 
374 transcription, signaling, nucleic acid metabolism or ion transport (Supplemental Table 4), 375 with only little fraction (15 individual miRNA-mRNA interactions) with strong validation 376 proofs available so far.

377 We also analysed whether the shared targets among four and five miRNAs (19 transcripts 378 collectively) build a network; for this, we used the STRING program ${ }^{58,59}$. STRING 379 database aims to collect, score and integrate all publicly available sources of protein380 protein interaction information, and to complement these with computational predictions. 381 Its goal is to achieve a comprehensive and objective global network, including direct epidermal growth factor receptor EGFR. Although, MAP3K3 is validated as a weak target (physical) as well as indirect (functional) interactions ${ }^{59}$. The resulting interaction network revealed that few mutual interactions could be detected (data not shown). In order to integrate the overlapping targets into a potential regulatory network, we added 17 of the most interacting potential targets, so called "hub genes": three for every six analyzed miRNAs (see Materials and Methods) (Supplemental Table 5). One potential target BTRC (F-box/WD repeat-containing protein $1 \mathrm{~A})$ is a hub for two miRNAs, namely miR150 and miR-132. Curiously, CREB1 appeared to be not only the shared target among miR-22 ${ }^{60}$, miR-13861, miR-14162, and miR-15057, but also acts as one of the most interacting genes targeted by miR-13861. In total, 11 out of 19 shared targets made no direct links, but the other eight targets could be directly combined into the hub-based network (Supplemental Figure 3); for instance, MAP3K3 kinase by interaction to of miR-145, there is a good chance for strong and physiologically relevant interaction as EGFR, in turn, interacts with VEGFA (vascular endothelial growth factor) that is also targeted by miR-145. In addition, MAP3K3 and EGFR are predicted targets of miR-141 (Supplemental Table 4), suggesting a functionally meaningful network comprised out of 
miRNAs and mRNAs. Eight out of 17 hub targets are validated by strong methods (e.g., SIRT1 as a target of miR-22 ${ }^{63}$ or SMAD2 as a target for miR-14564) and numerous mutual 400 links were found in this group regardless of the present level of their validation. Many hub genes encode either transcription factors (e.g., SDAM2) or components of the 402 ubiquitination machinery of proteins destined for degradation (e.g., KLHL11). The observation goes hand-in-hand with profound changes required for cells to make a global 404 change of their properties when switch phenotype occurs. Not surprisingly, therefore, that many components of such regulatory network may be targeted by numerous miRNAs to ensure high fidelity and efficiency of the response.

\section{Drug targeting of miRNA-mediated regulatory networks}

As the switch phenotype from the contractile to synthetic underlines numerous vascular pathologies, the pharmacological targeting of the key regulators could be a promising option to increase the therapeutic success. Consequently, we utilized the Drug-Gene Interaction Database (DGldb) (http://www.dgidb.org/) to predict the candidate drugs for

412 the targets of six miRNAs identified in this study (Fig. 4). Initially, we concentrated to find 413 the drugs that influence the activity of the hub targets; and indeed we found that 14 out of 41417 hub targets can be linked to approved or experimental drugs. Not surprisingly, one and 415 the same target can be influenced by several drugs. For instance, dasatinib and 416 gemcitabine could be potentially used as a cocktail to efficiently inhibit the activity of EGFR 417 (Fig. 5).

418 On the other hand, some of these drugs may be effective on several hub proteins 419 simultaneously. For example, Erlotinib could be effective on EGFR ${ }^{65}$ and $\mathrm{CBL}^{66}$, both 420 regulated by miR-141, or resveratrol on TP53 ${ }^{67}$ and SIRT1 ${ }^{68}$, both targeted by miR-22 
(Fig. 5). This type of drug-target interactions resemble pleiotropic activity of miRNAs and

422 may be of particular interest if a given miRNA has a limited regulatory activity upon other

423 processes, but switch phenotype. Ideally, a cocktail of drugs could be designed to inhibit

424 key targets of the selected miRNA. Potentially, a lower dose of the individual drug can be

425 used in such a mixture in order to achieve the desired effect, thereby, reducing unspecific

426 and side effects. Knowledge on miRNAs-mRNA interactions within a particular context

427 may serve as recipes for disease tailored or even personalized drug multiplexing.

\section{Acknowledgements}

429 We thank Jürgen Beneke, Nina Beil, and Dr. Margund Bach (Bioquant, Heidelberg 430 University) for the support with robotics and reagents, and confocal microscopy. And we 431 also thank Dr. Prama Pallavi, Ervins Zheng, Feng Guo, and Kaixuan Yan (Chirurgische 432 Klinik and European Center of Angioscience (ECAS) of Medical Faculty Mannheim, 433 Heidelberg University) for cooperation in the experiments.

\section{Funding}

436 This work was supported by RNA-CODE (Grant No. 031A298) of the German Federal 437 Ministry of Education and Research (BMBF), "Methoden für die Lebenswissenschaften" 438 of the Baden-Württemberg Stiftung (Grant No. P-LS-SPII/11), and the Scholarship for J. 439 Zhang of China Scholarship Council (CSC No. 2017-3109-A1).

\section{Author contributions}

441 J.Zhang designed and performed the experiments. J.Zhang, M.Keese, H.Erfle, and 442 V.Starkuviene planned the experiments, discussed the results, and wrote the manuscript. 
443 Z.Wang cooperated in the experiments. M.Gunkel and C.Sticht helped on the application 444 of KNIME software and analysis of the miRNA sequencing data, respectively. K.Kan 445 helped on analysis of the bioinformatics. N. Rahbari, and N.Gretz reviewed the manuscript. 


\section{References}

1 Brown, I. A. M. et al. Vascular Smooth Muscle Remodeling in Conductive and Resistance Arteries in Hypertension. Arterioscler Thromb Vasc Biol 38, 1969-1985, doi:10.1161/atvbaha.118.311229 (2018).

2 Virani, S. S. et al. Heart Disease and Stroke Statistics-2020 Update: A Report From the American Heart Association. Circulation 141, e139-e596, doi:10.1161/cir.0000000000000757 (2020).

3 Frismantiene, A., Philippova, M., Erne, P. \& Resink, T. J. Smooth muscle cell-driven vascular diseases and molecular mechanisms of VSMC plasticity. Cellular signalling 52, 48-64, doi:10.1016/j.cellsig.2018.08.019 (2018).

4 Davis-Dusenbery, B. N., Wu, C. \& Hata, A. Micromanaging vascular smooth muscle cell differentiation and phenotypic modulation. Arterioscler Thromb Vasc Biol 31, 2370-2377, doi:10.1161/ATVBAHA.111.226670 (2011).

5 Chistiakov, D. A., Orekhov, A. N. \& Bobryshev, Y. V. Vascular smooth muscle cell in atherosclerosis. Acta Physiol (Oxf) 214, 33-50, doi:10.1111/apha.12466 (2015).

6 Ayoubi, S., Sheikh, S. P. \& Eskildsen, T. V. Human induced pluripotent stem cell-derived vascular smooth muscle cells: differentiation and therapeutic potential. Cardiovasc Res 113, 1282-1293, doi:10.1093/cvr/cvx125 (2017).

7 Zhang, M. J. et al. An overview of potential molecular mechanisms involved in VSMC phenotypic modulation. Histochem Cell Biol 145, 119-130, doi:10.1007/s00418-015-1386-3 (2016).

8 Zhang, Y. N. et al. Phenotypic switching of vascular smooth muscle cells in the 'normal region' of aorta from atherosclerosis patients is regulated by miR-145. Journal of cellular and molecular medicine 20, 1049-1061, doi:10.1111/jcmm.12825 (2016).

9 Wei, X., Hou, X., Li, J. \& Liu, Y. miRNA-181a/b Regulates Phenotypes of Vessel Smooth Muscle Cells Through Serum Response Factor. DNA Cell Biol 36, 127-135, doi:10.1089/dna.2016.3525 (2017).

10 Ackers-Johnson, M. et al. Myocardin regulates vascular smooth muscle cell inflammatory activation and disease. Arterioscler Thromb Vasc Biol 35, 817-828, doi:10.1161/ATVBAHA.114.305218 (2015).

11 Cecchettini, A., Rocchiccioli, S., Boccardi, C. \& Citti, L. Vascular smooth-muscle-cell activation: proteomics point of view. Int Rev Cell Mol Biol 288, 43-99, doi:10.1016/B978-0-12-3860415.00002-9 (2011).

12 Huntzinger, E. \& Izaurralde, E. Gene silencing by microRNAs: contributions of translational repression and mRNA decay. Nat Rev Genet 12, 99-110, doi:10.1038/nrg2936 (2011).

13 Cheng, Y. et al. MicroRNA-145, a novel smooth muscle cell phenotypic marker and modulator, controls vascular neointimal lesion formation. Circ Res 105, 158-166, doi:10.1161/CIRCRESAHA.109.197517 (2009).

14 Yamaguchi, S. et al. The role of microRNA-145 in human embryonic stem cell differentiation into vascular cells. Atherosclerosis 219, 468-474, doi:10.1016/j.atherosclerosis.2011.09.004 (2011).

15 Huang, S. C. et al. Mir-22-3p Inhibits Arterial Smooth Muscle Cell Proliferation and Migration and Neointimal Hyperplasia by Targeting HMGB1 in Arteriosclerosis Obliterans. Cellular physiology and biochemistry : international journal of experimental cellular physiology, biochemistry, and pharmacology 42, 2492-2506, doi:10.1159/000480212 (2017).

16 Afzal, T. A. et al. NCK Associated Protein 1 Modulated by miRNA-214 Determines Vascular Smooth Muscle Cell Migration, Proliferation, and Neointima Hyperplasia. J Am Heart Assoc 5, doi:10.1161/JAHA.116.004629 (2016).

17 Li, P. et al. MicroRNA-663 regulates human vascular smooth muscle cell phenotypic switch and vascular neointimal formation. Circ Res 113, 1117-1127, doi:10.1161/CIRCRESAHA.113.301306 (2013). 
18 Cordes, K. R. et al. miR-145 and miR-143 regulate smooth muscle cell fate and plasticity. Nature 460, 705-710, doi:10.1038/nature08195 (2009).

19 Quintavalle, M., Elia, L., Condorelli, G. \& Courtneidge, S. A. MicroRNA control of podosome formation in vascular smooth muscle cells in vivo and in vitro. J Cell Biol 189, 13-22, doi:10.1083/jcb.200912096 (2010).

20 Elia, L. et al. The knockout of miR-143 and -145 alters smooth muscle cell maintenance and vascular homeostasis in mice: correlates with human disease. Cell Death Differ 16, 1590-1598, doi:10.1038/cdd.2009.153 (2009).

21 Zhao, H. et al. MicroRNA-22 regulates smooth muscle cell differentiation from stem cells by targeting methyl CpG-binding protein 2. Arterioscler Thromb Vasc Biol 35, 918-929, doi:10.1161/atvbaha.114.305212 (2015).

22 Steffen, A. et al. Sra-1 and Nap1 link Rac to actin assembly driving lamellipodia formation. The EMBO Journal 23, 749-759, doi:10.1038/sj.emboj.7600084 (2004).

23 Yang, F. et al. miR-22 Is a Novel Mediator of Vascular Smooth Muscle Cell Phenotypic Modulation and Neointima Formation. Circulation 137, 1824-1841, doi:10.1161/CIRCULATIONAHA.117.027799 (2018).

24 Khachigian, L. M. Transcription Factors Targeted by miRNAs Regulating Smooth Muscle Cell Growth and Intimal Thickening after Vascular Injury. International journal of molecular sciences 20, doi:10.3390/ijms20215445 (2019).

25 Farina, F. M. et al. miR-128-3p Is a Novel Regulator of Vascular Smooth Muscle Cell Phenotypic Switch and Vascular Diseases. Circ Res 126, e120-e135, doi:10.1161/CIRCRESAHA.120.316489 (2020).

26 Peng, W., Li, T., Pi, S., Huang, L. \& Liu, Y. Suppression of circular RNA circDHCR24 alleviates aortic smooth muscle cell proliferation and migration by targeting miR-149-5p/MMP9 axis. Biochemical and biophysical research communications 529, 753-759, doi:10.1016/j.bbrc.2020.06.067 (2020).

27 Ye, D. et al. MicroRNA125amediated regulation of the mevalonate signaling pathway contributes to high glucoseinduced proliferation and migration of vascular smooth muscle cells. Mol Med Rep 22, 165-174, doi:10.3892/mmr.2020.11077 (2020).

28 Zhang, L. et al. High-Throughput RNAi Screening Identifies a Role for the Osteopontin Pathway in Proliferation and Migration of Human Aortic Smooth Muscle Cells. Cardiovascular drugs and therapy 30, 281-295, doi:10.1007/s10557-016-6663-4 (2016).

29 Bulkescher, R., Starkuviene, V. \& Erfle, H. Solid-phase reverse transfection for intracellular delivery of functionally active proteins. Genome Res 27, 1752-1758, doi:10.1101/gr.215103.116 (2017).

30 Chang, S. et al. Phenotypic modulation of primary vascular smooth muscle cells by short-term culture on micropatterned substrate. PLoS One 9, e88089, doi:10.1371/journal.pone.0088089 (2014).

31 Zhao, S. et al. QuickMIRSeq: a pipeline for quick and accurate quantification of both known miRNAs and isomiRs by jointly processing multiple samples from microRNA sequencing. BMC Bioinformatics 18, 180, doi:10.1186/s12859-017-1601-4 (2017).

32 Ritchie, M. E. et al. limma powers differential expression analyses for RNA-sequencing and microarray studies. Nucleic Acids Res 43, e47, doi:10.1093/nar/gkv007 (2015).

$33 \mathrm{Gu}$, Z., Eils, R. \& Schlesner, M. Complex heatmaps reveal patterns and correlations in multidimensional genomic data. Bioinformatics (Oxford, England) 32, 2847-2849, doi:10.1093/bioinformatics/btw313 (2016).

34 Sticht, C., De La Torre, C., Parveen, A. \& Gretz, N. miRWalk: An online resource for prediction of microRNA binding sites. PLoS One 13, e0206239, doi:10.1371/journal.pone.0206239 (2018).

35 Cotto, K. C. et al. DGIdb 3.0: a redesign and expansion of the drug-gene interaction database. Nucleic Acids Res 46, D1068-D1073, doi:10.1093/nar/gkx1143 (2018).

36 Chin, C. H. et al. cytoHubba: identifying hub objects and sub-networks from complex interactome. BMC Syst Biol 8 Suppl 4, S11, doi:10.1186/1752-0509-8-S4-S11 (2014). 


\begin{tabular}{|c|c|c|}
\hline 43 & 37 & $\begin{array}{l}\text { Lee, H. S. et al. Prostaglandin D2 stimulates phenotypic changes in vascular smooth muscle cells. } \\
\text { Experimental \& molecular medicine 51, 1-10, doi:10.1038/s12276-019-0330-3 (2019). }\end{array}$ \\
\hline 5 & 38 & Nanjare, M., Kuo, F. \& Gerecht, S. Derivation and maturation of synthetic and contractile vascular \\
\hline 6 & & $\begin{array}{l}\text { nooth muscle cells from human pluripotent stem cells. Cardiovasc Res 97, 321-330, } \\
\text { i: } 10.1093 / \text { cvr/cys } 315(2013) \text {. }\end{array}$ \\
\hline 48 & 39 & i, Y. et al. Direct labeling and visualization of blood vessels with lipophilic carbocyanine dye Dil. \\
\hline 49 & & Vat Protoc 3, 1703-1708, doi:10.1038/nprot.2008.172 (2008). \\
\hline 50 & 40 & Erfle, H. et al. Work Flow for Multiplexing siRNA Assays by Solid-Phase Reverse Transfection in \\
\hline 51 & & $\begin{array}{l}\text { Multiwell Plates. Journal of Biomolecular Screening 13, 575-580, doi:10.1177/1087057108320133 } \\
\text { (2008). }\end{array}$ \\
\hline 53 & 41 & $\begin{array}{l}\text { Erfle, H. \& Pepperkok, R. Arrays of transfected mammalian cells for high content screening } \\
\text { microscopy. Methods Enzymol 404, 1-8, doi:10.1016/S0076-6879(05)04001-2 (2005). }\end{array}$ \\
\hline 5 & 42 & $\begin{array}{l}\text { Erfle, H., Simpson, J. C., Bastiaens, P. I. \& Pepperkok, R. siRNA cell arrays for high-content screening } \\
\text { microscopy. Biotechniques 37, 454-458, 460, 462, doi:10.2144/04373RT01 (2004). }\end{array}$ \\
\hline 57 & 43 & $\begin{array}{l}\text { Chen, M., Shen, C., Zhang, Y. \& Shu, H. MicroRNA-150 attenuates hypoxia-induced excessive } \\
\text { proliferation and migration of pulmonary arterial smooth muscle cells through reducing HIF-1a } \\
\text { expression. Biomed Pharmacother } 93,861-868 \text {, doi:10.1016/j.biopha.2017.07.028 (2017). }\end{array}$ \\
\hline 60 & 44 & $\begin{array}{l}\text { Feng, S. et al. MiR-93 regulates vascular smooth muscle cell proliferation, and neointimal } \\
\text { formation through targeting Mfn2. Int J Biol Sci 15, 2615-2626, doi:10.7150/ijbs.36995 (2019). }\end{array}$ \\
\hline 62 & 15 & iu, Y. et al. MiR-138 suppresses airway smooth muscle cell proliferation through the PI3K/AKT \\
\hline 63 & & signaling pathway by targeting PDK1. Exp Lung Res 41, 363-369, \\
\hline 564 & & doi:10.3109/01902148.2015.1041581 (2015). \\
\hline 65 & 46 & $\begin{array}{l}\text { Guo, X. et al. miRNA-145 inhibits VSMC proliferation by targeting CD40. Scientific Reports 6, 35302, } \\
\text { doi:10.1038/srep35302 (2016). }\end{array}$ \\
\hline $\begin{array}{l}567 \\
568\end{array}$ & 47 & $\begin{array}{l}\text { Xie, C. et al. KOBAS 2.0: a web server for annotation and identification of enriched pathways and } \\
\text { diseases. Nucleic Acids Res 39, W316-322, doi:10.1093/nar/gkr483 (2011). }\end{array}$ \\
\hline $\begin{array}{l}569 \\
570\end{array}$ & 48 & $\begin{array}{l}\text { Zhou, Y. et al. Metascape provides a biologist-oriented resource for the analysis of systems-level } \\
\text { datasets. Nat Commun 10, 1523, doi:10.1038/s41467-019-09234-6 (2019). }\end{array}$ \\
\hline 571 & 49 & Zhang, X., Chen, J. \& Wang, S. Serum Amyloid A Induces a Vascular Smooth Muscle Cell Phenotype \\
\hline $\begin{array}{l}572 \\
573\end{array}$ & & $\begin{array}{l}\text { Switch through the p38 MAPK Signaling Pathway. BioMed Research International 2017, 4941379, } \\
\text { doi:10.1155/2017/4941379 (2017). }\end{array}$ \\
\hline 574 & 50 & Song, S. H. et al. Fibroblast Growth Factor 12 Is a Novel Regulator of Vascular Smooth Muscle Cell \\
\hline $\begin{array}{l}575 \\
576\end{array}$ & & $\begin{array}{l}\begin{array}{l}\text { Plasticity and Fate. Arterioscler } \\
\text { doi:10.1161/ATVBAHA.116.308017 (2016). }\end{array} \\
\text { Thromb Vasc Biol 36, 1928-1936, }\end{array}$ \\
\hline $\begin{array}{l}577 \\
578\end{array}$ & 51 & $\begin{array}{l}\text { Garat, C. V., Crossno, J. T., Jr., Sullivan, T. M., Reusch, J. E. \& Klemm, D. J. Inhibition of } \\
\text { phosphatidylinositol 3-kinase/Akt signaling attenuates hypoxia-induced pulmonary artery }\end{array}$ \\
\hline 79 & & remodeling and suppresses CREB depletion in arterial smooth muscle cells. J Cardiovasc Pharmacol \\
\hline 580 & & 62, 539-548, doi:10.1097/fjc.0000000000000014 (2013). \\
\hline 581 & 52 & Balint, B. et al. Collectivization of Vascular Smooth Muscle Cells via TGF- $\beta$-Cadherin-11-Dependent \\
\hline $\begin{array}{l}582 \\
583\end{array}$ & & $\begin{array}{l}\begin{array}{l}\text { Adhesive Switching. Arterioscler } \\
\text { doi:10.1161/atvbaha.115.305310 (2015). }\end{array} \\
\text { Thromb }\end{array}$ \\
\hline 584 & 53 & Lin, C. S. et al. The CCL5/CCR5 Axis Promotes Vascular Smooth Muscle Cell Proliferation and \\
\hline $\begin{array}{l}585 \\
586 \\
587\end{array}$ & & $\begin{array}{l}\text { Atherogenic Phenotype Switching. Cellular physiology and biochemistry: international journal of } \\
\text { experimental cellular physiology, biochemistry, and pharmacology 47, 707-720, } \\
\text { doi:10.1159/000490024 (2018). }\end{array}$ \\
\hline $\begin{array}{l}588 \\
589 \\
590\end{array}$ & 54 & $\begin{array}{l}\text { Michel, J. B., Jondeau, G. \& Milewicz, D. M. From genetics to response to injury: vascular smooth } \\
\text { muscle cells in aneurysms and dissections of the ascending aorta. Cardiovasc Res 114, 578-589, } \\
\text { doi:10.1093/cvr/cvy006 (2018). }\end{array}$ \\
\hline
\end{tabular}


55 Li, S., Sims, S., Jiao, Y., Chow, L. H. \& Pickering, J. G. Evidence from a novel human cell clone that adult vascular smooth muscle cells can convert reversibly between noncontractile and contractile phenotypes. Circ Res 85, 338-348 (1999).

56 Zhu, X. et al. miR-145 sensitizes ovarian cancer cells to paclitaxel by targeting Sp1 and Cdk6. Int J Cancer 135, 1286-1296, doi:10.1002/ijc.28774 (2014).

57 Guo, Y. H. et al. Wnt/ $\beta$-catenin pathway transactivates microRNA-150 that promotes EMT of colorectal cancer cells by suppressing CREB signaling. Oncotarget 7, 42513-42526, doi:10.18632/oncotarget.9893 (2016).

58 Jensen, L. J. et al. STRING 8--a global view on proteins and their functional interactions in 630 organisms. Nucleic Acids Res 37, D412-416, doi:10.1093/nar/gkn760 (2009).

59 Szklarczyk, D. et al. STRING v11: protein-protein association networks with increased coverage, supporting functional discovery in genome-wide experimental datasets. Nucleic Acids Res 47, D607-d613, doi:10.1093/nar/gky1131 (2019).

60 Friedrich, M. et al. CREB1 is affected by the microRNAs miR-22-3p, miR-26a-5p, miR-27a-3p, and miR-221-3p and correlates with adverse clinicopathological features in renal cell carcinoma. Scientific Reports 10, 6499, doi:10.1038/s41598-020-63403-y (2020).

61 Zhang, C. et al. MicroRNA-138 modulates glioma cell growth, apoptosis and invasion through the suppression of the AKT/mTOR signalling pathway by targeting CREB1. Oncology reports 44, 25592568, doi:10.3892/or.2020.7809 (2020).

62 Liu, T. et al. SPION-mediated miR-141 promotes the differentiation of HuAESCs into dopaminergic neuron-like cells via suppressing IncRNA-HOTAIR. Journal of cellular and molecular medicine 22, 2299-2310, doi:10.1111/jcmm.13512 (2018).

63 Ming, G. F., Wu, K., Hu, K., Chen, Y. \& Xiao, J. Corrigendum to "NAMPT regulates senescence, proliferation, and migration of endothelial progenitor cells through the SIRT1 AS IncRNA/miR22/SIRT1 pathway". Biochemical and biophysical research communications 524, 523-524, doi:10.1016/j.bbrc.2020.02.111 (2020).

64 Li, L., Mao, D., Li, C. \& Li, M. miR-145-5p Inhibits Vascular Smooth Muscle Cells (VSMCs) Proliferation and Migration by Dysregulating the Transforming Growth Factor-b Signaling Cascade. Med Sci Monit 24, 4894-4904, doi:10.12659/MSM.910986 (2018).

65 Wen, P. Y. et al. Phase I/II study of erlotinib and temsirolimus for patients with recurrent malignant gliomas: North American Brain Tumor Consortium trial 04-02. Neuro-oncology 16, 567-578, doi:10.1093/neuonc/not247 (2014).

66 Kadera, B. E. et al. Low expression of the E3 ubiquitin ligase CBL confers chemoresistance in human pancreatic cancer and is targeted by epidermal growth factor receptor inhibition. Clin Cancer Res 21, 157-165, doi:10.1158/1078-0432.Ccr-14-0610 (2015).

$67 \mathrm{Xu}, \mathrm{R}$. Y. et al. Resveratrol attenuates myocardial hypoxia/reoxygenation-induced cell apoptosis through DJ-1-mediated SIRT1-p53 pathway. Biochemical and biophysical research communications 514, 401-406, doi:10.1016/j.bbrc.2019.04.165 (2019).

68 Caon, I. et al. Sirtuin 1 reduces hyaluronan synthase 2 expression by inhibiting nuclear translocation of NF-KB and expression of the long-noncoding RNA HAS2-AS1. J Biol Chem 295, 3485-3496, doi:10.1074/jbc.RA119.011982 (2020). 

serum concentrations in the growth media. A. Increase of $\alpha$-SMA in HAoVSMCs in low-serum media conditions. B. HAoVSMCs showed reduced migration in the contractile phenotype (1\% FCS) and increased migration in the synthetic phenotype (10\% FCS).

C. Quantification of the gap closure.

Fig. 2 Cell shape segmentation by KNIME software and the ratio of con / syn after miRNAs

641 transfection. A. Automated detection of contractile and synthetic phenotypes. Contractile phenotype is 642 indicated by red arrows and the synthetic phenotype is indicated by yellow arrows. B. Quantification of the 643 phenotypic switch of HAoVSMCs after miRNA transfection. Compared with the control group, the 644 transfection groups (miR-22, miR-145, miR-214, and miR-663a) appear to have significantly higher ratios of 645 con / syn at $48 \mathrm{~h}$ and $72 \mathrm{~h} .{ }^{*} p<0.05,{ }^{* *} p<0.01$.

646 Fig. 3 Differential expression of miRNAs in the contractile phenotype comparing with the control 647 group. A. Heatmap of each replicate, indicating closeness between these groups and the difference 648 between them. Red color indicates high expression of miRNAs, and green color indicates low expression of 649 miRNAs. N: normal serum, L: low serum. B. Volcano plot shows that the individual up-regulated and down650 regulated miRNAs after averaging replicates of the group with the contractile phenotype and the control 651 group. Red dots indicate the upregulated miRNAs, and green dots represent downregulated miRNAs. The 652 thresholds are: upregulated miRNAs $\left(\log _{2} \mathrm{FC}>0.6, \mathrm{FC}>1.5, p<0.05\right)$, downregulated miRNAs $\left(\log _{2} \mathrm{FC}\right.$ $653<-0.6, \mathrm{FC}<2 / 3, p<0.05)$. C. Overlap between the hit miRNAs derived from microscopy-based screening 654 and sequencing. 
Fig. 1

A

B C

$\alpha$-SMA

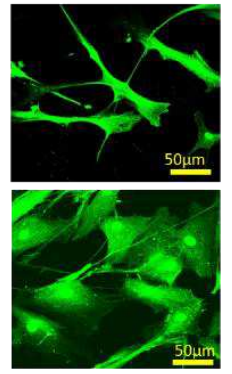

Ctrl

Synthetic

$1 \%$ FCS

Contractile

$\mathbf{O h}$
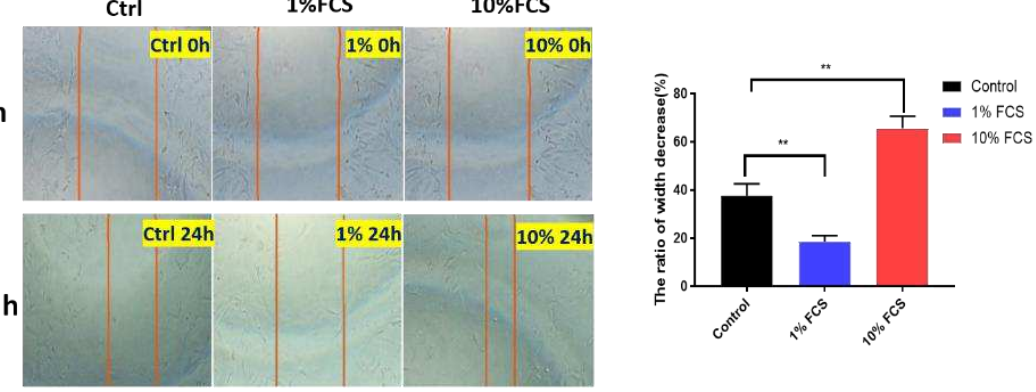
Fig. 2

A

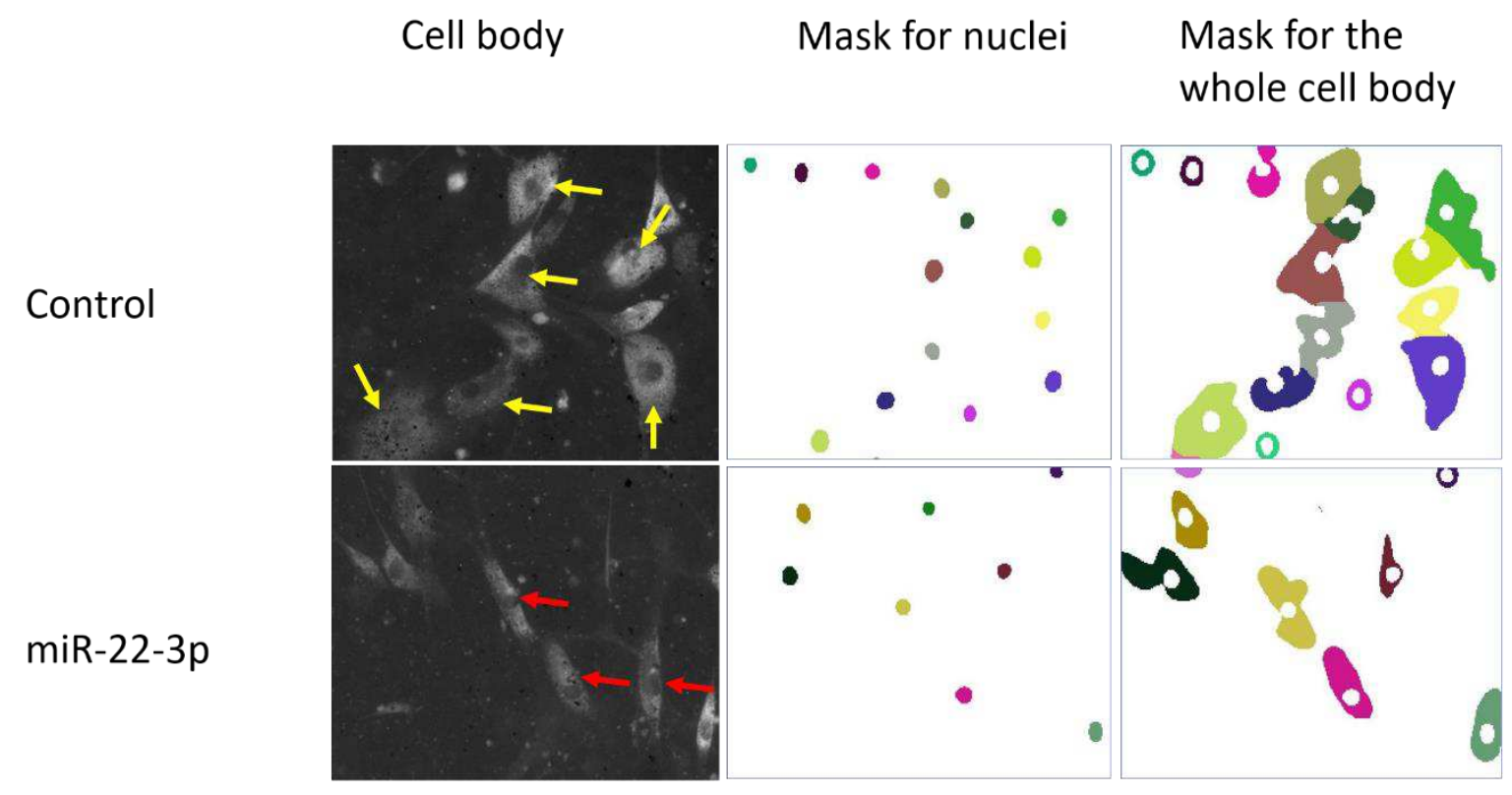

B

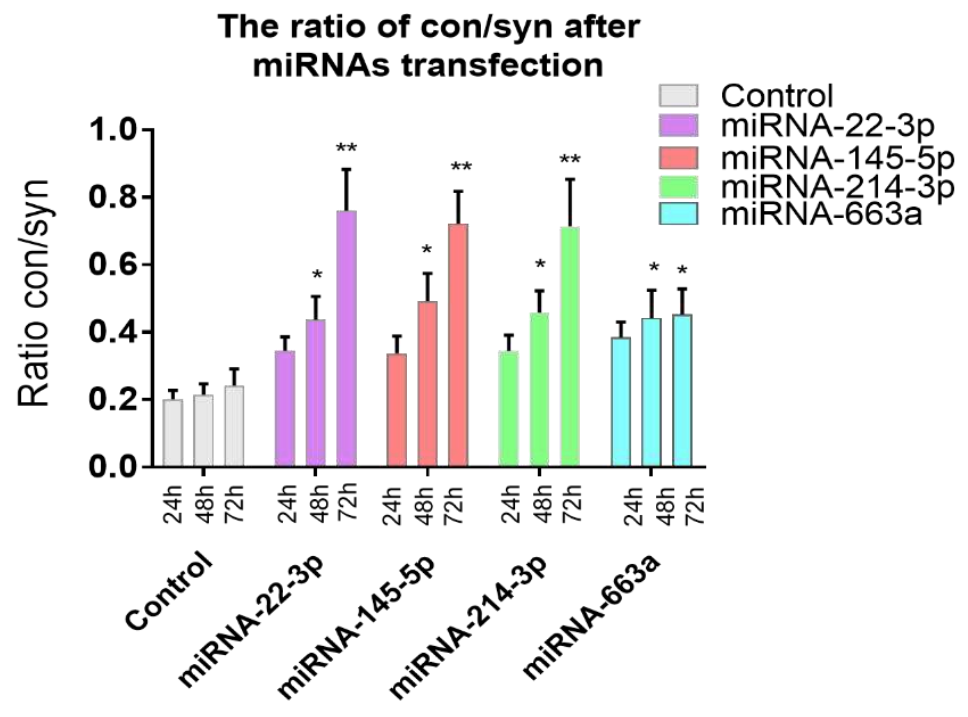


Fig. 3

A
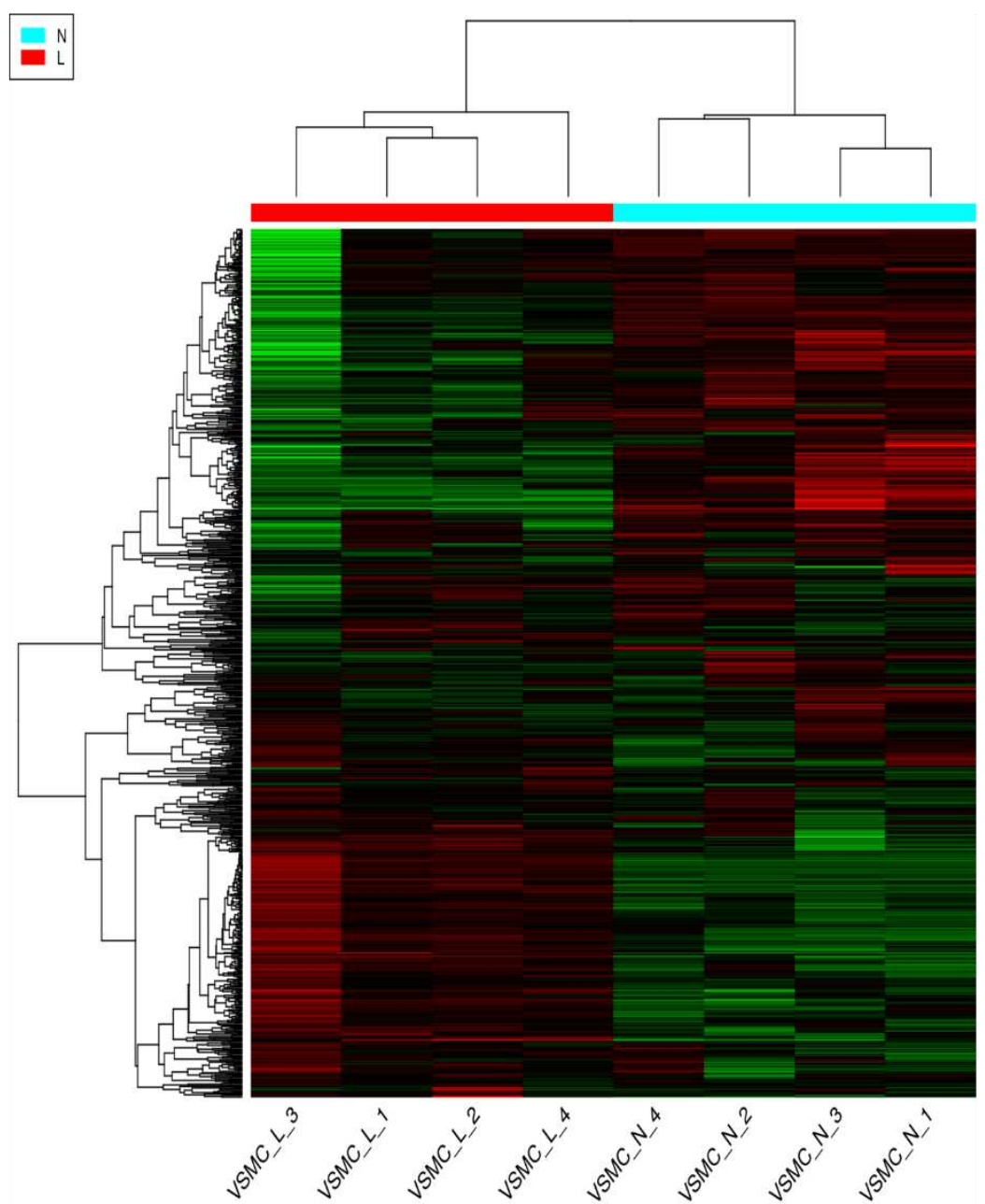

Color Key

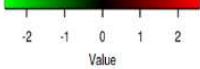

B

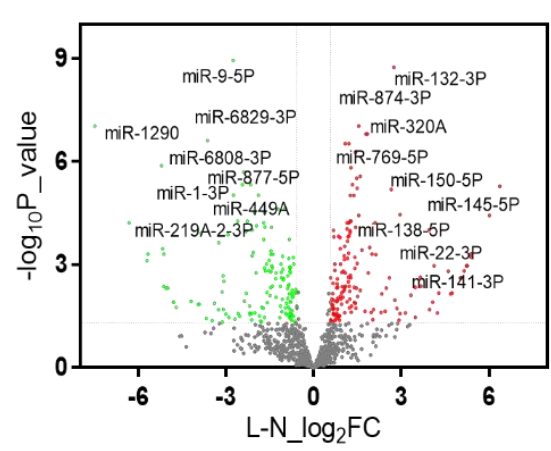

C

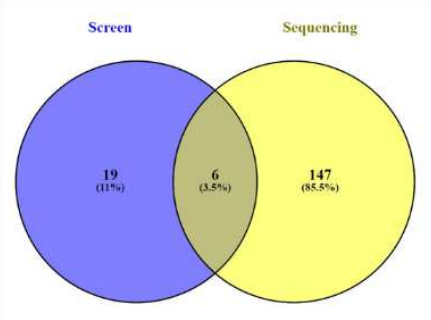

The overlapped six miRNAs:

miR-132-3p, miR-138-5p, miR-141-3p, miR-145-5p, miR-150-5p, and miR-22-3p 
Fig. 4

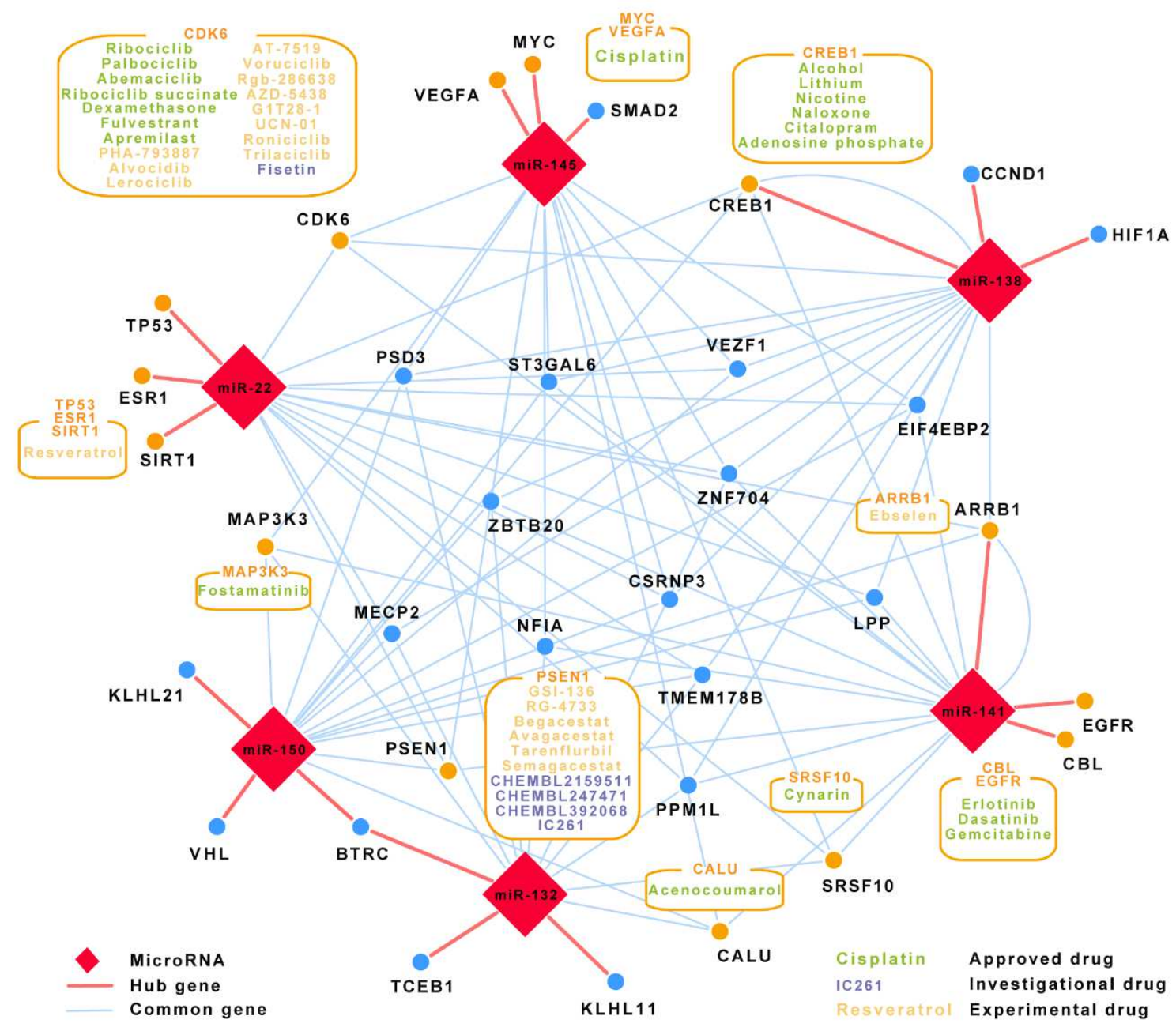


Fig. 5

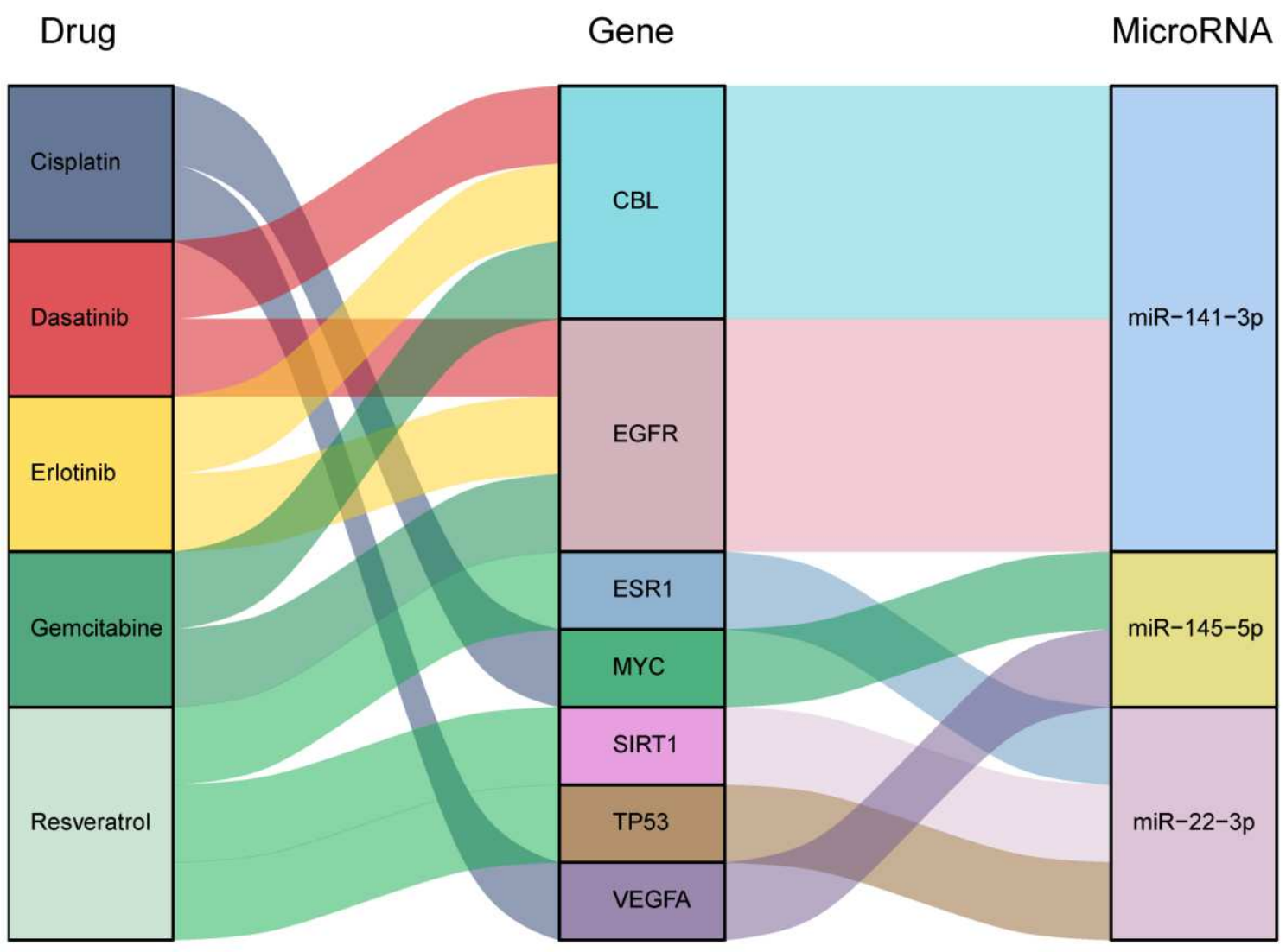




\section{Figures}

Fig. 1

A

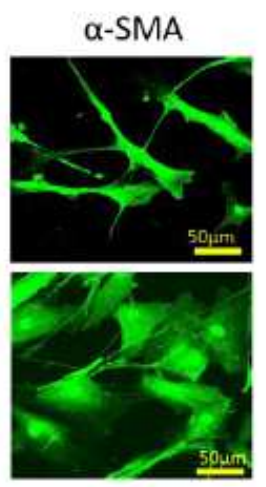

B

$1 \%$ FCS

Contractile

Ctrl

Synthetic

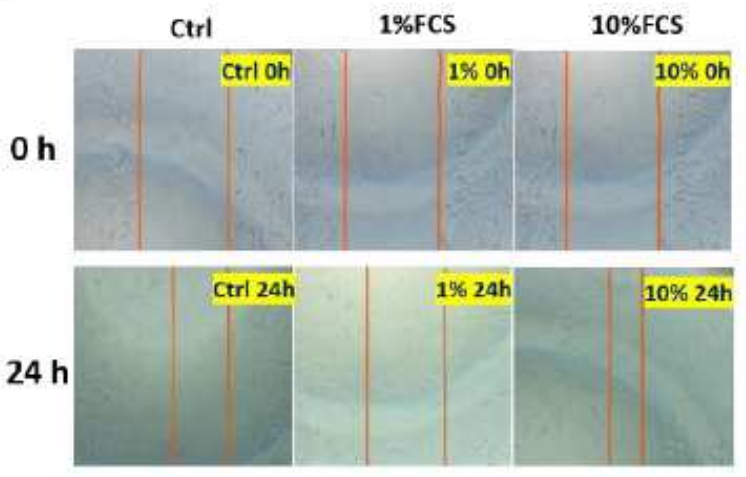

C

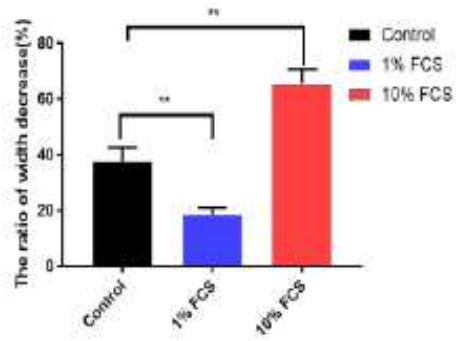

\section{Figure 1}

Validation of the contractile and synthetic phenotypes of HAoVSMCs induced by varying serum concentrations in the growth media. A. Increase of a-SMA in HAoVSMCs in low-serum media conditions. B. HAoVSMCs showed reduced migration in the contractile phenotype (1\% FCS) and increased migration in the synthetic phenotype (10\% FCS). C. Quantification of the gap closure. 
Fig. 2

A

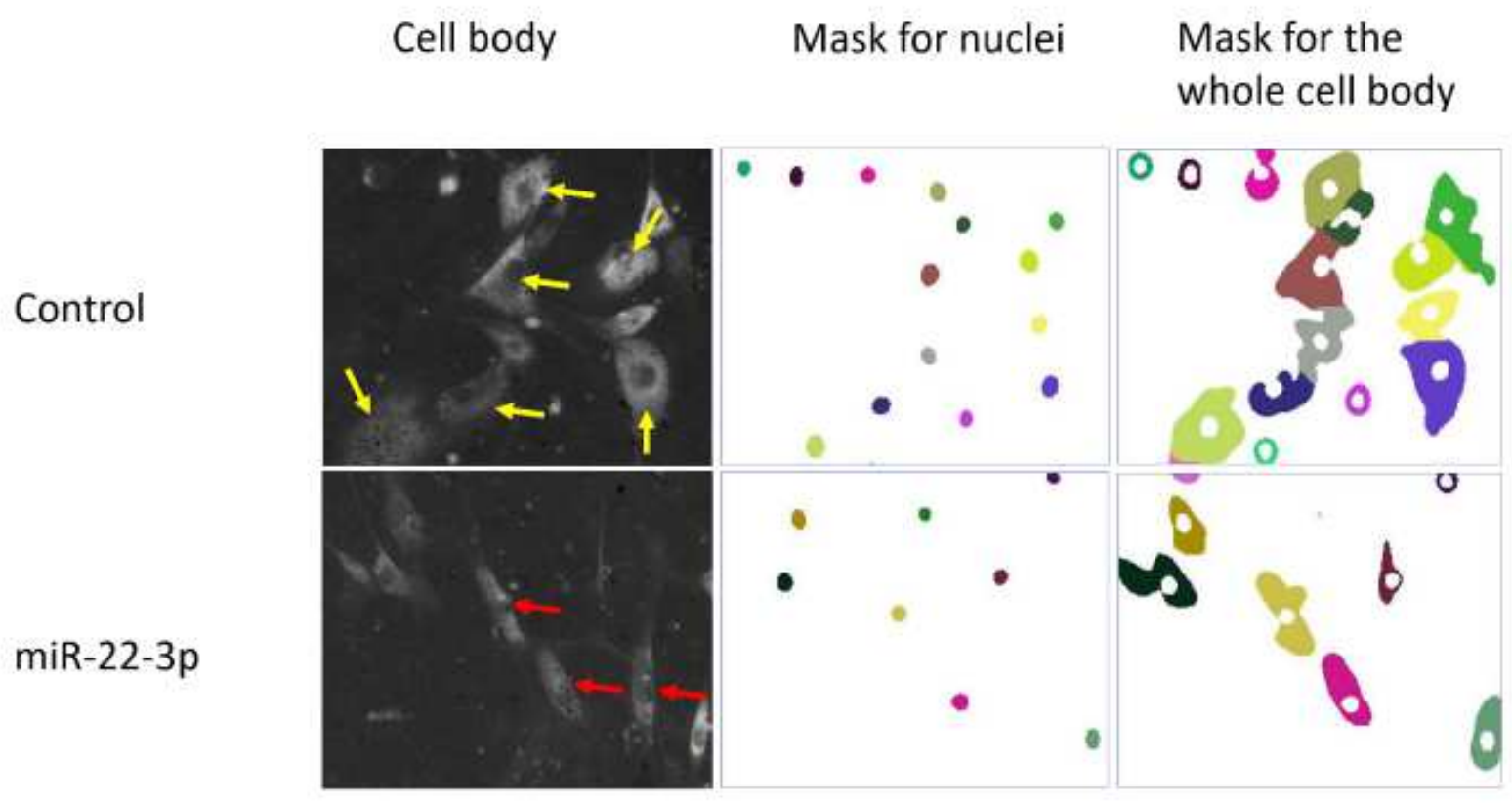

B

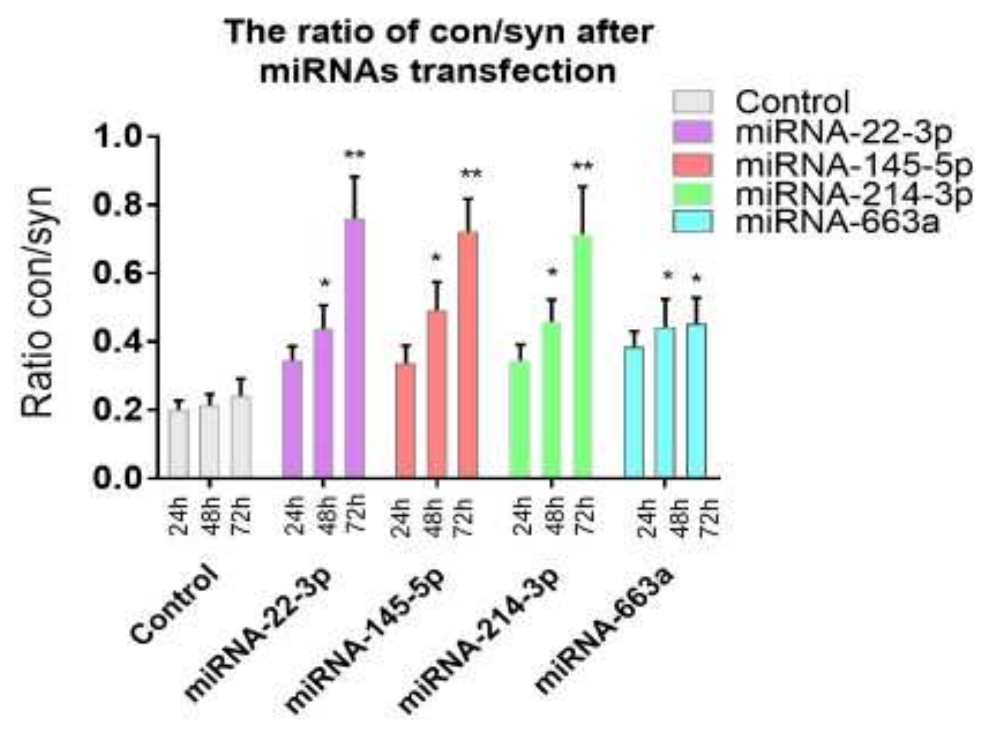

Figure 2

Cell shape segmentation by KNIME software and the ratio of con / syn after miRNAs transfection. A. Automated detection of contractile and synthetic phenotypes. Contractile phenotype is indicated by red arrows and the synthetic phenotype is indicated by yellow arrows. B. Quantification of the phenotypic switch of HAoVSMCs after miRNA transfection. Compared with the control group, the transfection groups 
(miR-22, miR-145, miR-214, and miR-663a) appear to have significantly higher ratios of con / syn at $48 \mathrm{~h}$ and $72 \mathrm{~h} . * \mathrm{p}<0.05, * * \mathrm{p}<0.01$.

Fig. 3

A
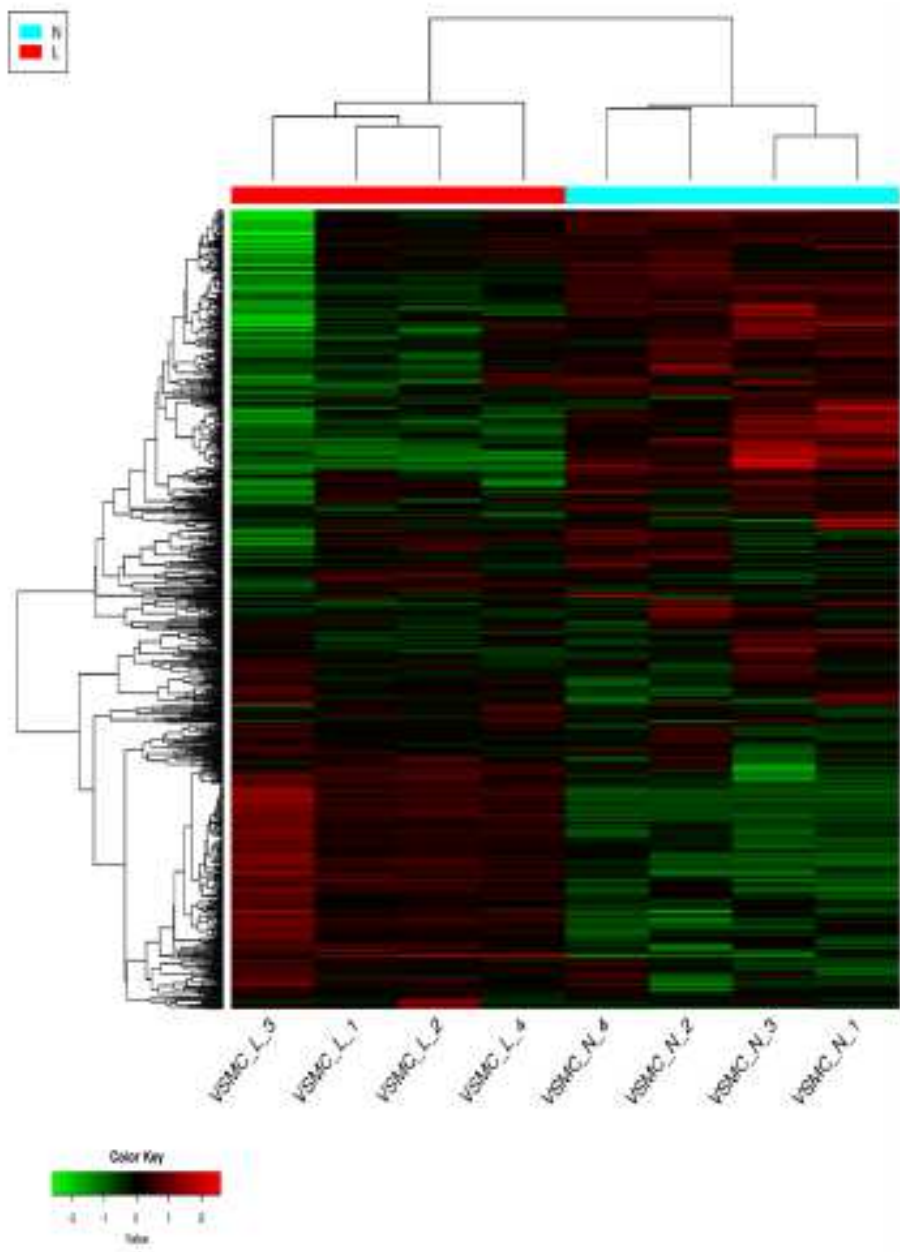

B

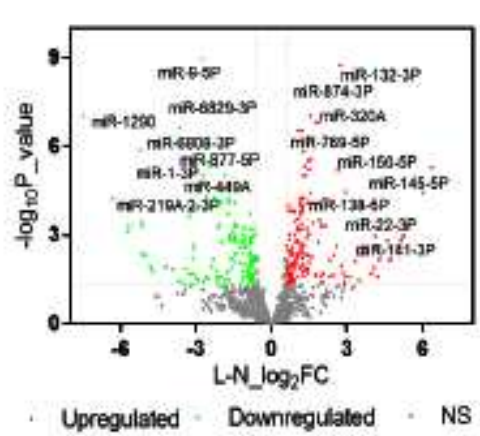

C

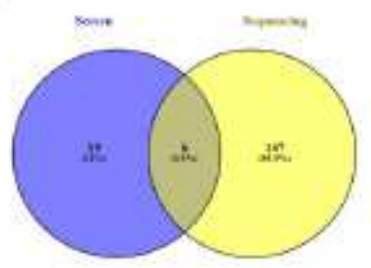

The overlapped six miRNAs:

miR-132-3p, miR-138-5p, miR-141-3p, miR-145-5p, miR-150-5p, and miR-22-3p

\section{Figure 3}

Differential expression of miRNAs in the contractile phenotype comparing with the control group. A. Heatmap of each replicate, indicating closeness between these groups and the difference between them. Red color indicates high expression of miRNAs, and green color indicates low expression of miRNAs. N: normal serum, L: low serum. B. Volcano plot shows that the individual up-regulated and down-regulated miRNAs after averaging replicates of the group with the contractile phenotype and the control group. Red 
dots indicate the upregulated miRNAs, and green dots represent downregulated miRNAs. The thresholds are: upregulated miRNAs (Log2FC > 0.6, FC > 1.5, $p<0.05$ ), downregulated miRNAs (Log2FC < -0.6, FC < $2 / 3, p<0.05)$. C. Overlap between the hit miRNAs derived from microscopy-based screening and sequencing.

Fig. 4

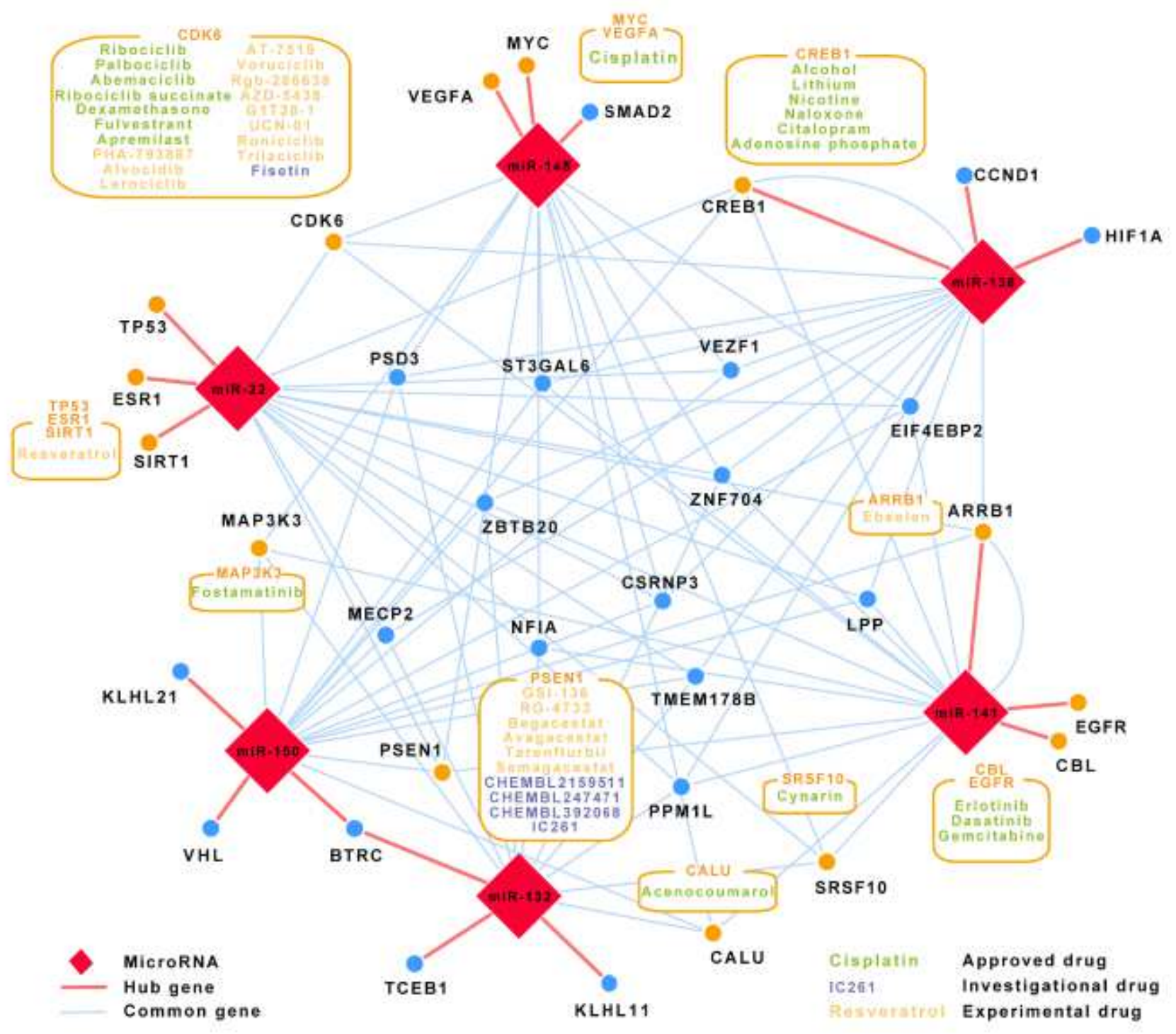

Figure 4

Predicted drugs affecting hub targets and the common targets among four and five miRNAs. 
Fig. 5

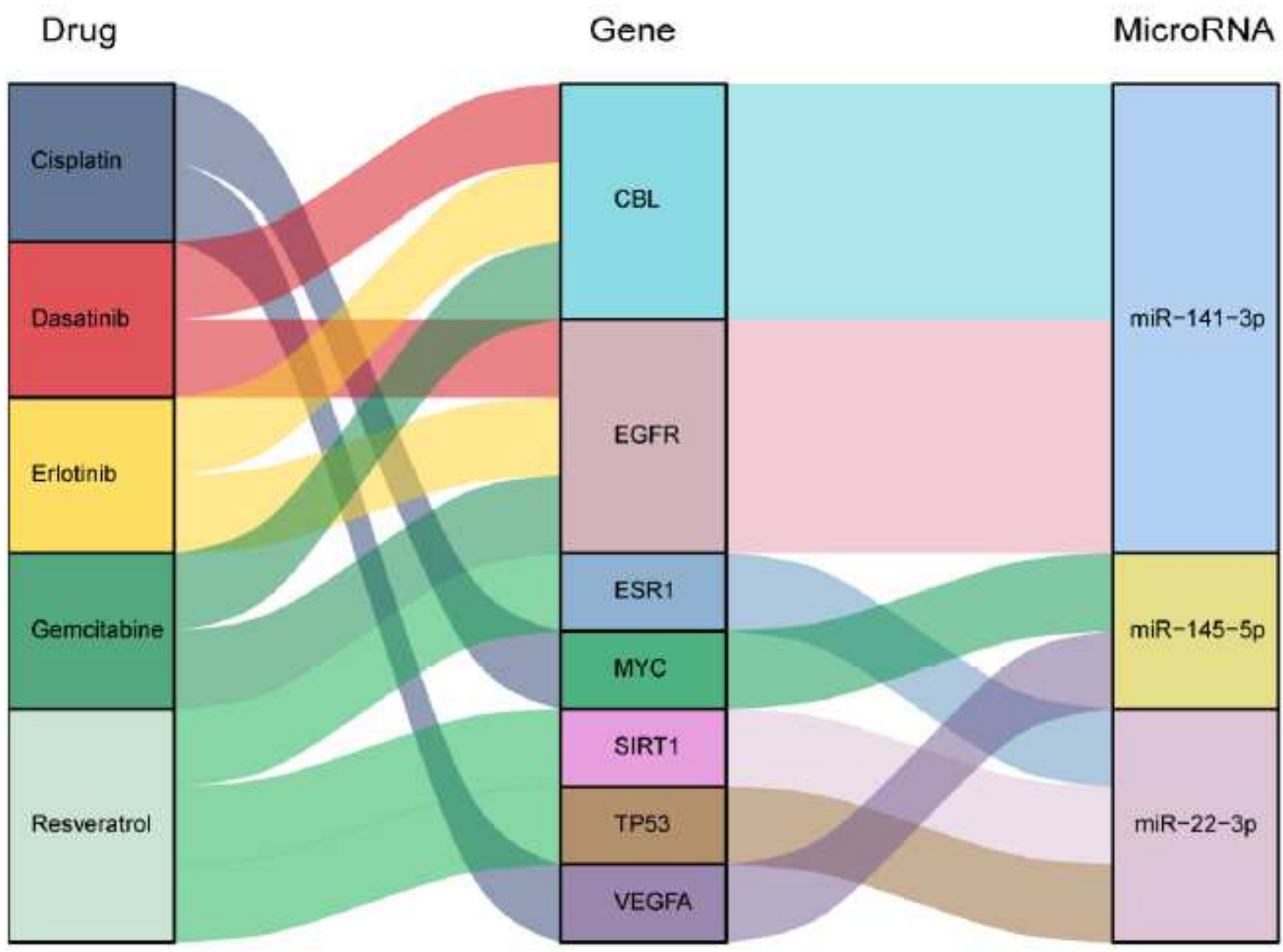

Figure 5

Subset of the combinatorial interactions among miRNAs-targets-drugs.

\section{Supplementary Files}

This is a list of supplementary files associated with this preprint. Click to download.

- Supplements20210508MKeese.pdf 